\title{
ARTÍ́CULO DE INVESTIGACIÓN
}

\section{Distribución de foraminíferos bentónicos (Protozoa: Foraminiferida) en la ensenada Quillaipe ( $41^{\circ} 32^{\prime}$ S; $\left.72^{\circ} 44^{\prime} \mathrm{O}\right)$, Chile: Implicaciones para el estudio del nivel del mar}

\author{
Distribution of benthic foraminifera (Protozoa: Foraminiferida) in the Quillaipe Inlet

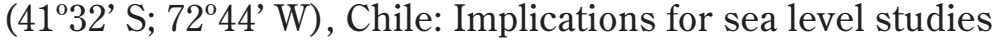

LEONARDO D. FERNÁNDEZ11, * \& JAIME A. ZAPATA ${ }^{2}$

${ }^{1}$ Centro de Estudios en Biodiversidad (CEBCh), Magallanes 1979, Osorno, Chile

${ }^{2}$ Departamento de Ciencias Básicas, Universidad de Los Lagos, Casilla 933, Osorno, Chile

*Autor correspondiente: limnoleo@gmail.com

\begin{abstract}
RESUMEN
Los foraminíferos de las marismas salobres son utilizados como indicadores del cambio producido en el nivel del mar durante el Holoceno. No obstante, los foraminíferos de las marismas de Chile han sido escasamente estudiados por lo que se desconoce la factibilidad de utilizarlos para este fin. Para subsanar esto se desarrolló una investigación en el intermareal de la ensenada de Quillaipe, Chile, con los objetivos de determinar los foraminíferos y su distribución vertical; analizar los parámetros que explican su distribución y determinar las especies que son útiles como indicadoras del nivel del mar. Los resultados revelaron 18 especies (nueve aglutinadas y nueve calcáreas) distribuidas en dos grandes Zonas (I y II). La Zona I se restringió a la parte más alta y vegetada del intermareal (marisma) y estuvo habitada solo por taxa aglutinados. Aquí se registraron bajos valores de diversidad $\left(\mathrm{H}^{\prime}=0.567\right), \mathrm{pH}(6.6)$ y salinidad (18.7) y un dominio de la especie aglutinada Haplophragmoides manilaensis. En cambio, la Zona II se limitó a la zona menos elevada y sin vegetación del intermareal (llanura de marea) y estuvo habitada por una comunidad calcárea-aglutinada. Contrariamente, los valores de diversidad $\left(\mathrm{H}^{\prime}=0.909\right)$, $\mathrm{pH}(7.7)$ y salinidad (32.8) fueron más altos, y la especie dominante fue la calcárea Ammonia beccarii. Por otro lado, la asociación aglutinada Trochamminita salsa-Jadammina macrescens se restringió a la parte más alta de la marisma y a los valores más bajos de salinidad y pH. Estos antecedentes permiten concluir que la distribución de los foraminíferos está controlada por la salinidad, el pH y la elevación del intermareal y que la asociación Trochamminita salsa-Jadammina macrescens son indicadoras del máximo nivel del mar en la ensenada de Quillaipe.
\end{abstract}

Palabras clave: Chile, distribución vertical, foraminíferos, marisma salobre, nivel del mar.

\section{ABSTRACT}

Saltmarsh foraminifera are used as indicators of sea-level change which occurs during the Holocene. In Chile however, the saltmarsh foraminifera have been poorly studied, so it is unknown the feasibility of using them for this purpose. To address this issue, a research was conducted in the intertidal Quillaipe Inlet, Chile, in order to determine the vertical distribution of foraminifera and to analyze the parameters that explain their distribution and determine the species that are useful as indicators of sea level. The results revealed 18 species (nine calcareous and nine agglutinated) distributed in two zones (I and II). Zone I was restricted to the saltmarsh and inhabited exclusively by agglutinated community. Here, diversity $\left(\mathrm{H}^{\prime}=0.567\right), \mathrm{pH}(6.6)$ and salinity (18.7) values were low with a dominance of agglutinated species Haplophragmoides manilaensis. Zone II was restricted to tidal flat and inhabited by a calcareous-agglutinated community. In contrast, diversity $\left(\mathrm{H}^{\prime}=0.909\right), \mathrm{pH}(7.7)$ and salinity (32.8) values were higher, and the dominant species was the calcareous Ammonia beccarii. On the other hand, the agglutinated association Trochamminita salsa-Jadammina macrescens was restricted to the top of the saltmarsh and to low salinity and $\mathrm{pH}$ values. We conclude that the distribution of foraminifera is controlled by salinity, $\mathrm{pH}$ and the elevation of intertidal area and suggest that Trochamminita salsa-Jadammina macrescens association could be used as indicators of the maximum sea-level in the Quillaipe Inlet.

Key words: Chile, foraminifera, saltmarsh, sea-level, vertical distribution.

\section{INTRODUCCIÓN}

Los foraminíferos que habitan las marismas salobres presentan una distribución vertical estrechamente relacionada con la elevación y el régimen mareal de estos ambientes (Horton et al. 1999). Esta característica permite identificar con mucha precisión el límite entre el ambiente terrestre y el marino (Edwards \& Horton 2006). Por esta razón, los foraminíferos están siendo 
muy utilizados para reconstruir los cambios producidos en el nivel del mar durante el último periodo glacial (Holoceno) y en consecuencia predecir cómo fluctuarán producto del calentamiento global (e.g., Scott \& Medioli 1978, 1980a, 1980b, Scott \& Leckie 1990, Hayward et al. 1999, Horton \& Edwards 2003, Scott et al. 2003, Patterson et al. 2004, Horton \& Edwards 2006, Scott 2006, Pascual \& RodríguezLázaro 2006, Horton \& Murray 2007, Horton \& Culver 2008, Leorri et al. 2008) .

La metodología utilizada para estas reconstrucciones es conocida como "función de transferencia” y se desarrolla comparando estadísticamente la distribución vertical de la comunidad moderna y fósil de estos organismos. No obstante, es condición que la distribución de la comunidad moderna responda a algún gradiente ambiental y que su composición permanezca similar en el registro fósil (Berkeley et al. 2007). Por lo mismo, la reconstrucción de los niveles del mar en cualquier sitio requiere de un previo conocimiento de la distribución moderna de estos organismos (Horton et al. 1999, Horton \& Edwards 2003, Horton \& Murray 2007, Vázquez et al. 2007).

Un gran número de trabajos han estudiado la distribución de los foraminíferos modernos en las marismas salobres del Atlántico Norte (e.g., Le Campion 1970, Phleger 1970, Pujos 1976, Scott \& Medioli 1978, 1980a, Smith et al. 1984, Murray 1991, Gehrels 1994a, 1994b, De Rijk 1995, De Rijk \& Troelstra 1997, Cearreta et al. 2002, Moreno et al. 2005, Woodroffe et al. 2005, Horton \& Edwards 2006, Fatela et al. 2007, Horton \& Edwards 2006, Horton \& Murray 2007, Horton \& Culver 2008, Leorri et al. 2008), del Atlántico Sur (e.g., Cusminski et al. 2009) y del Pacífico Norte (e.g., Williams 1989, Patterson 1990, Jennings \& Nelson 1992, Guilbault et al. 1995, 1996, Scott et al. 1996), lo que en algunos casos ha permitido la posterior aplicación de la "función de transferencia".

Muchas de estas investigaciones han determinado que la distribución vertical de los foraminíferos es influenciada por varios factores locales, destacando la salinidad (De Rijk 1995, De Rijk \& Troelstra 1997, Fatela et al. 2007) o el pH (Woodroffe et al. 2005) como los más importantes. Sin embargo, otros estudios han concluido que la distribución vertical de estos organismos está más bien controlada por la elevación y el régimen mareal de estos ambientes (e.g., Cearreta et al. 2002, Edwards \& Horton 2006, Horton \& Edwards 2006, Horton \& Murray 2007), siendo esta la conclusión más aceptada y la base del desarrollo y aplicación de la "función de transferencia”.

Si bien existe una amplia cantidad de información respecto a este tema, es difícil aseverar si esta metodología es aplicable a las marismas templadas del Pacífico Sur, ya que, son escasos los trabajos que han investigado la distribución de estos organismos en estos ambientes, existiendo para Chile solo el trabajo de Jennings et al. (1995), quienes sugirieron que la distribución vertical de los foraminíferos está controlada por la zonación floral de la marisma y el de Fernández \& Zapata (2010), en donde estudiaron preliminarmente la influencia del pH sobre la distribución de un foraminífero calcáreo. La conclusión del primer trabajo no es consistente con resultados obtenidos en investigaciones recientes y el segundo se centra exclusivamente en una especie.

Estos antecedentes evidencian la necesidad de investigar en las marismas la distribución moderna de estos organismos, con tal de generar información de línea base actual que compruebe la factibilidad de aplicar en un futuro la "función de transferencia".

En este sentido este trabajo tiene por objetivos determinar los foraminíferos y su distribución vertical local en una marisma del Pacífico Sur; analizar los parámetros que explican la distribución y determinar preliminarmente las especies que podrían ser útiles para reconstruir los cambios producidos en el nivel del mar durante el Holoceno.

\section{MÉTODOS}

La ensenada Quillaipe $\left(41^{\circ} 32^{\prime} \mathrm{S} ; 72^{\circ} 44^{\prime} \mathrm{O}\right)$ está localizada a $18 \mathrm{~km}$ de la ciudad de Puerto Montt, en la parte oriental del seno de Reloncaví, Región de Los Lagos, Chile. Posee un intermareal de $2500 \mathrm{~m}$ de longitud dividido en: a) una zona vegetada (marisma) ubicada en la parte alta del intermareal y b) una zona sin vegetación (llanura de marea) que continúa inmediatamente después de la zona vegetada, en dirección hacia el mar (Fig. 1). El día del muestreo se registró una pleamar máxima de $5.91 \mathrm{~m}$ y una bajamar mínima de $1.50 \mathrm{~m}$, correspondiendo el régimen mareal al tipo mixto (SHOA 2007).

En el intermareal se muestreó, en una oportunidad (otoño del 2007), un total de 20 estaciones distribuidas en una transecta de $1700 \mathrm{~m}$ de longitud. El estudio no 
se centró únicamente en la marisma, ya que, un solo ambiente no entrega una analogía apropiada para el registro fósil (Horton et al. 1999), junto con permitir la discusión con trabajos similares. Por lo tanto, las primeras seis estaciones (1-6) se ubicaron en $300 \mathrm{~m}$ de la marisma y las siguientes (7-20) en $1400 \mathrm{~m}$ de la llanura de marea (Fig. 1).

En cada estación se extrajo con un core los $10 \mathrm{~cm}$ superiores del sedimento. Posteriormente, desde cada muestra, se retiraron tres submuestras correspondientes al primer, quinto y décimo centímetro (contando desde la superficie hacia abajo). Seguidamente, las tres submuestras de cada estación fueron depositadas en una bolsa rotulada, mezcladas y fijadas con etanol al $98 \%$.

Tradicionalmente la mayoría de las investigaciones consideran solo el centímetro superior del sedimento para estudios foraminiferológicos (e.g., Debenay et al. 1998, Alve \& Murray 1999, Horton et al. 1999, Murray \& Alve 1999, Gehrels et al. 2001, Horton et al. 2003, Edwards et al. 2004, Horton et al. 2005, Woodroffe et al. 2005). Esto puede provocar que las especies que
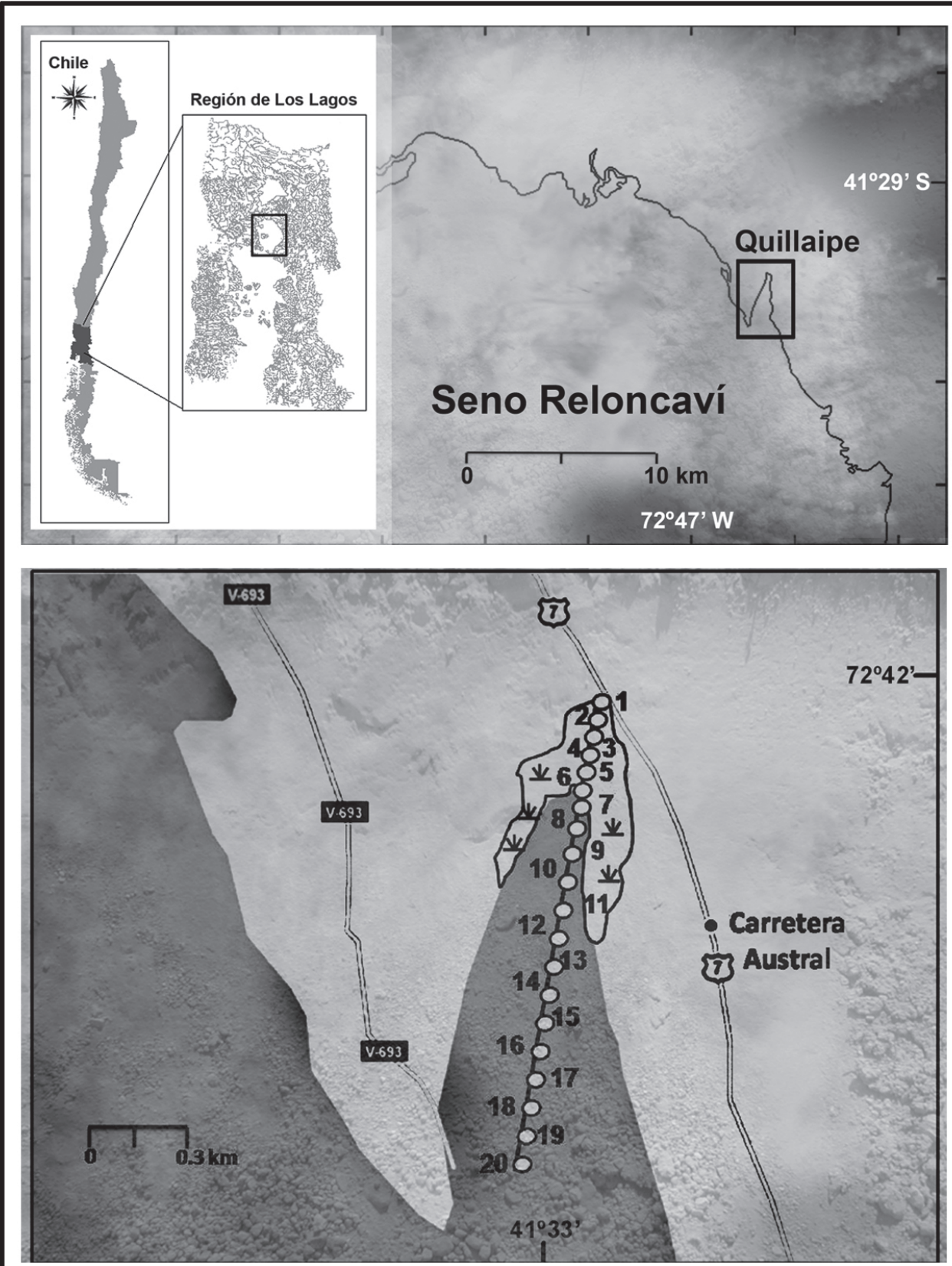

Fig. 1: Ubicación del área de estudio, transecta y estaciones en la ensenada Quillaipe.

Location of the study area, transect and stations in the Quillaipe Inlet. 
viven bajo el centímetro superior estén subrepresentadas o ausentes como resultado de esta estrategia de muestreo (Goldstein \& Harben 1993, Goldstein 1998), entregando una representación incompleta de la distribución de la comunidad moderna (Berkeley et al. 2007). En consecuencia, la profundidad y metodología de muestreo utilizada en este trabajo se consideró apropiada para registrar todas las especies (epipélicas y endopélicas) y para facilitar futuras comparaciones con el registro fósil, previniendo la subrepresentatividad y/o la ausencia de algunas especies.

Posteriormente, cada muestra fue depositada en un tamiz de $63 \mu \mathrm{m}$ de abertura de malla y lavada con agua corriente para retirar el exceso de fango y fijador, para luego, concentrarla en $15 \mathrm{~cm}^{3}$ de agua destilada. Desde alícuotas de cada muestra, examinadas bajo un microscopio estereoscópico binocular (40x), se extrajeron al azar los primeros 200 ejemplares de foraminíferos, duplicando la cantidad mínima sugerida por Saffert \& Thomas (1998). Los ejemplares extraídos correspondieron a la comunidad total (vivos más muertos), ya que es de mayor utilidad en la definición de biofacies (Patterson 1990), refleja el impacto de los procesos tafonómicos (Horton 1999, Murray 2000) y son una representación general más precisa de las condiciones ambientales, puesto que integran las fluctuaciones tanto estacionales como temporales (Horton \& Murray 2007).

Paralelamente se midió en cada estación la salinidad, el pH y la elevación (respecto al nivel de marea más bajo del día del muestreo), utilizando un termo-salinómetro 556 MPS, un peachímetro Hanna Instrument y el método de Emery (1961) y Krause (2004) respectivamente.

Basándose en la elevación de las estaciones y en el estudio florístico realizado previamente por San Martín \& Ramírez (2002), se dividió la marisma en tres zonas florales: a) marisma alta (MA), situada entre los 1.80$1.60 \mathrm{~m}$ y codominada por junquillos (Juncus balticus Will.) y totora azul (Scirpus americanus [Pers.]); b) marisma media (MM), ubicada entre los 1.60-1.35 m, con amplio dominio del pasto azul de marismas (Puccinellia glaucescens [Phil.]); y c) marisma baja (MB), extendida entre los 1.35-1.25 m, dominada por la pimpinela (Anagallis alternifolia Cav.) A esta zona floral le continuó la llanura de marea (zona sin vegetación) (LM), situada entre los 1.25-0 m.

Se determinó para cada estación la riqueza de especies (S), el índice de diversidad (H') de ShannonWiener (1963) y la(s) especie(s) dominante(s). Se estableció como especie dominante a toda aquella que presentó una abundancia $a \geq 25 \%$.

Se analizó la zonación vertical de los foraminíferos en la zona de estudio usando el índice de similitud de Jaccard (Saiz \& Avendaño 1976). Para expresar espacialmente las similitudes entre estaciones se construyó un dendrograma basado en el método del ligamento promedio que utiliza la media aritmética no ponderada (UPGMA) (Crisci \& López 1983). Todos los cálculos fueron realizados utilizando el software Biodiversity Pro v.2.

Para la determinación taxonómica hasta el nivel de género, se empleó la clasificación de Loeblich \& Tappan (1988) y para el nivel específico se recurrió principalmente a los trabajos de Boltovskoy \& Theyer (1970), Boltovskoy et al. (1980), Hayward et al. (1997), Hayward et al. 1999), Zapata \& Cea (2004) y Vázquez \& Patterson (2008). Las especies identificadas fueron fotografiadas con un Microscopio Electrónico de Barrido JEOL T-300 operando a $10 \mathrm{kv}$.

\section{RESULTADOS}

En un total de 4000 ejemplares recolectados en la ensenada de Quillaipe se identificaron 18 especies de foraminíferos bentónicos (Figs. 2 y 3). Dos especies aglutinadas (Haplophragmoides manilaensis Andersen, 1953 y Miliammina fusca [Brady, 1884]) y dos especies calcáreas (Ammonia beccarii [Linnaeus, 1758] y Elphidium williamsoni Haynes, 1973) dominaron la comunidad y representaron en conjunto más del $70 \%$ de la abundancia relativa total (Tabla 1 ).

Las especies aglutinadas Jadammina macrescens (Brady, 1870) y Trochamminita salsa (Cushman \& Brönnimann, 1948), no dominaron en ninguna estación pero su distribución se restringió exclusivamente a la marisma alta (MA) y parte de la marisma media (MM) (estaciones 1-2 y 1-3 respectivamente). Por el contrario, la especie aglutinada $M$. fusca se distribuyó en toda la ensenada de Quillaipe (estaciones 1-20) (Tabla 1).

El dendrograma que expresa las similitudes entre las estaciones permitió clasificar el área de estudio en dos zonas (I y II) (Fig. 4). La Zona I se distribuyó entre los 1.80 y $1.25 \mathrm{~m}$ de elevación y comprendió toda la marisma (estaciones 1-6) y se caracterizó por estar habitada solo por foraminíferos aglutinados. La especie más dominante en esta zona fue $H$. manilaensis y su abundancia relativa alcanzó hasta un 66 \% (Fig. 5). Por otro lado, la Zona II se distribuyó entre los 1.25 y 0 m de elevación y comprendió toda la llanura de marea (estaciones 7-20) y se caracterizó por estar codominada por especies aglutinadas y calcáreas. No obstante, se diferenció de la otra zona principalmente por tener altos porcentajes de los foraminíferos calcáreos $A$. beccarii y E. williamsoni (frecuencias relativas máximas de un 51 y $28 \%$ respectivamente) (Fig. 5).

Adicionalmente, considerando un $65 \%$ de afinidad, el dendrograma permitió identificar en la Zona I (marisma) y en la Zona II (llanura de marea) dos subzonas faunísticas verticales (IA, IB y IIA, IIB respectivamente) (Tabla 1). Estas subzonas estuvieron definidas por cambios proporcionales en las abundancias de las especies dominantes y no coincidieron siempre con la zonación floral de la marisma. 


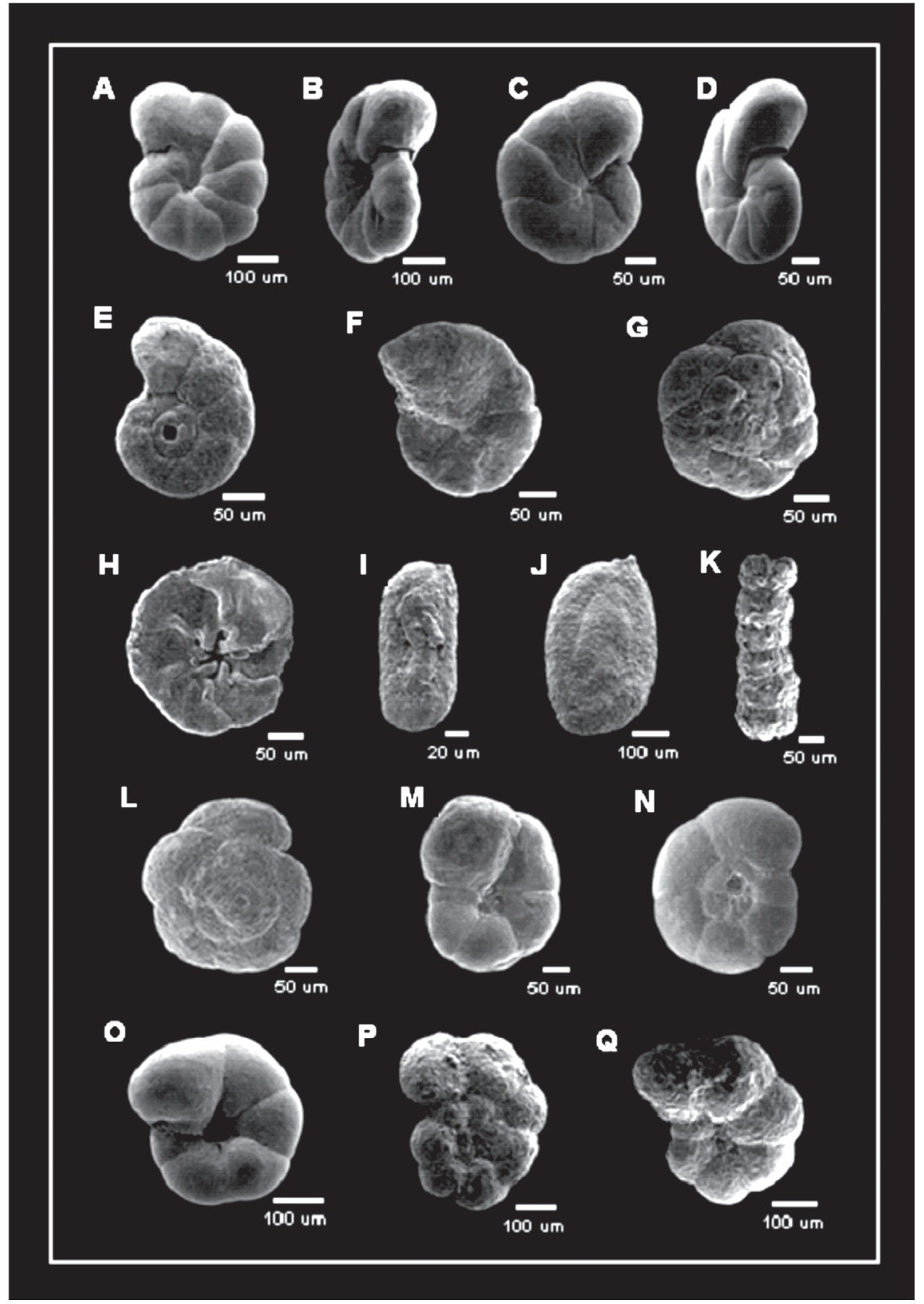

Fig. 2: Foraminíferos aglutinados de la ensenada Quillaipe: (A, B) Haplophragmoides manilaensis Andersen, 1953 (A) vista lateral, (B) vista látero-abertural; (C, D) Haplophragmoides wilberti Andersen, 1953 (C) vista lateral, (D) vista látero-abertural; (E, F) Jadammina macrescens (Brady, 1870) (E) vista dorsal, (F) vista ventral; (G, H) Lepidodeuterammina ochracea (Williamson, 1858) (G) vista dorsal, (H) vista ventral; (I, J) Miliammina fusca (Brady, 1870) ambas son vistas laterales; (K) Reophax moniliformis Siddall, 1886 vista lateral; (L, M) Tiphotrocha comprimata (Cushman \& Brönnimann, 1948) (L) vista dorsal, (M) vista ventral; (N, O) Trochammina inflata (Montagu, 1808) (N) vista dorsal, (O) vista ventral; (P, Q) Trochamminita salsa (Cushman \& Brönnimann, 1948) (P) vista dorsal, (Q) vista ventral.

Agglutinated foraminifera from Quillaipe Inlet: (A, B) Haplophragmoides manilaensis A) lateral view, B) lateral-apertural view; (C, D) Haplophragmoides wilberti (C) lateral view, (D) lateral-apertural view; (E, F) Jadammina macrescens (E) dorsal view, (F) ventral view; $(\mathrm{G}, \mathrm{H})$ Lepidodeuterammina ochracea (G) dorsal view, (H) ventral view; (I, J) Miliammina fusca both are lateral views; (K) Reophax moniliformis lateral view; (L, M) Tiphotrocha comprimata (L) dorsal view, (M) ventral view; $(\mathrm{N}, \mathrm{O})$ Trochammina inflata $(\mathrm{N})$ dorsal view, $(\mathrm{O})$ ventral view; $(\mathrm{P}, \mathrm{Q})$ Trochamminita salsa $(\mathrm{P})$ dorsal view, (Q) ventral view. 


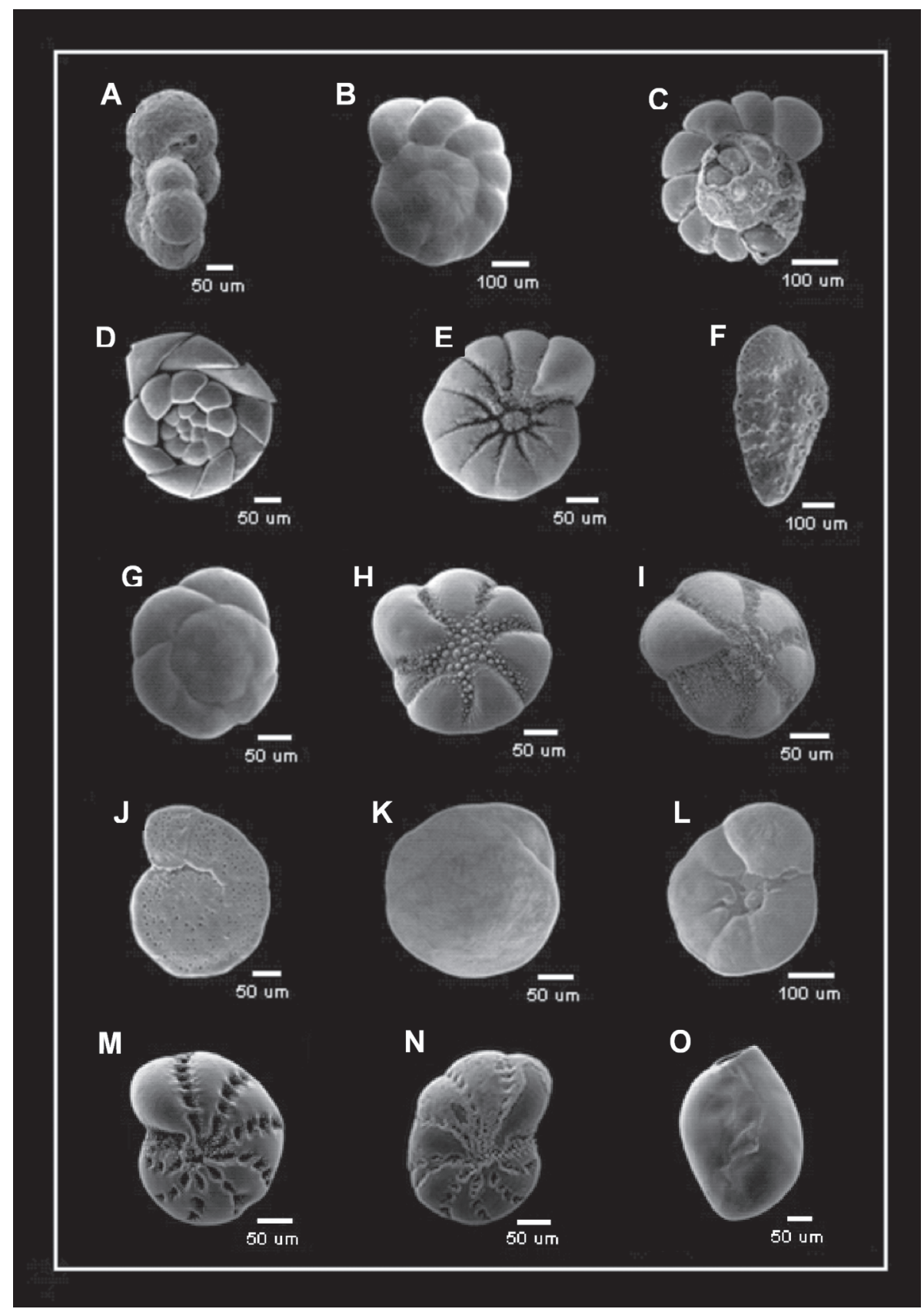

Fig. 3: Foraminíferos aglutinados y calcáreos de la Ensenada Quillaipe: (A) Trochamminita salsa (Cushman \& Brönnimann, 1948) vista abertural; (B-E) Ammonia beccarii (Linnaeus, 1758) (B) vista dorsal, (C, D) vista dorsal de ejemplares con disolución de la conchilla, (E) vista ventral; (F) Bolivina doniezi Cushman \& Wickenden, 1929 vista lateral; (G, H) Buccella frigida (Cushman, 1922) (G) vista dorsal, (H) vista ventral; (I) Buccella peruviana (Orbigny, 1839) vista ventral; (J) Cibicides aknerianus (Orbigny, 1846) vista dorsal; (K, L) Discorbis williamsoni Chapman \& Parr, 1932 (K) vista dorsal, (L) vista ventral; (M) Elphidium excavatum (Terquem, 1876) vista lateral; (N) Elphidium williamsoni Haynes, 1973 vista lateral; (O) Quinqueloculina seminula (Linnaeus, 1758) vista lateral.

Agglutinated and calcareous foraminifera from Quillaipe Inlet: (A) Trochamminita salsa apertural view; (B-E) Ammonia beccarii (B) dorsal view, (C, D) dorsal view of specimens with shell dissolution, (E) ventral view; (F) Bolivina doniezi lateral view; (G, H) Buccella frigida (Cushman) (G) dorsal view, (H) ventral view; (I) Buccella peruviana ventral view; (J) Cibicides aknerianus dorsal view; (K, L) Discorbis williamsoni (K) dorsal view, (L) ventral view; (M) Elphidium excavatum lateral view; (N) Elphidium williamsoni lateral view; (O) Quinqueloculina seminula lateral view. 


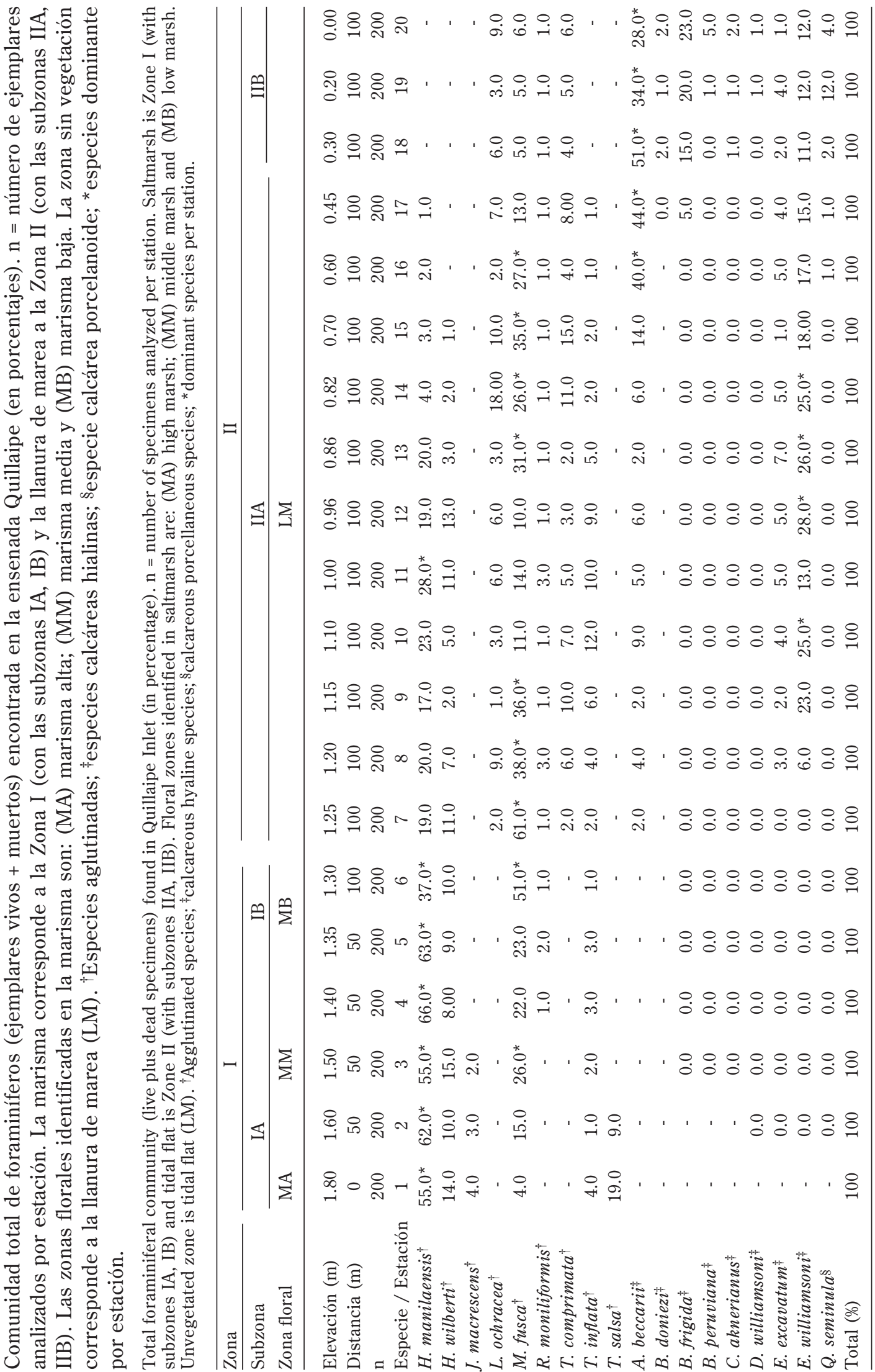




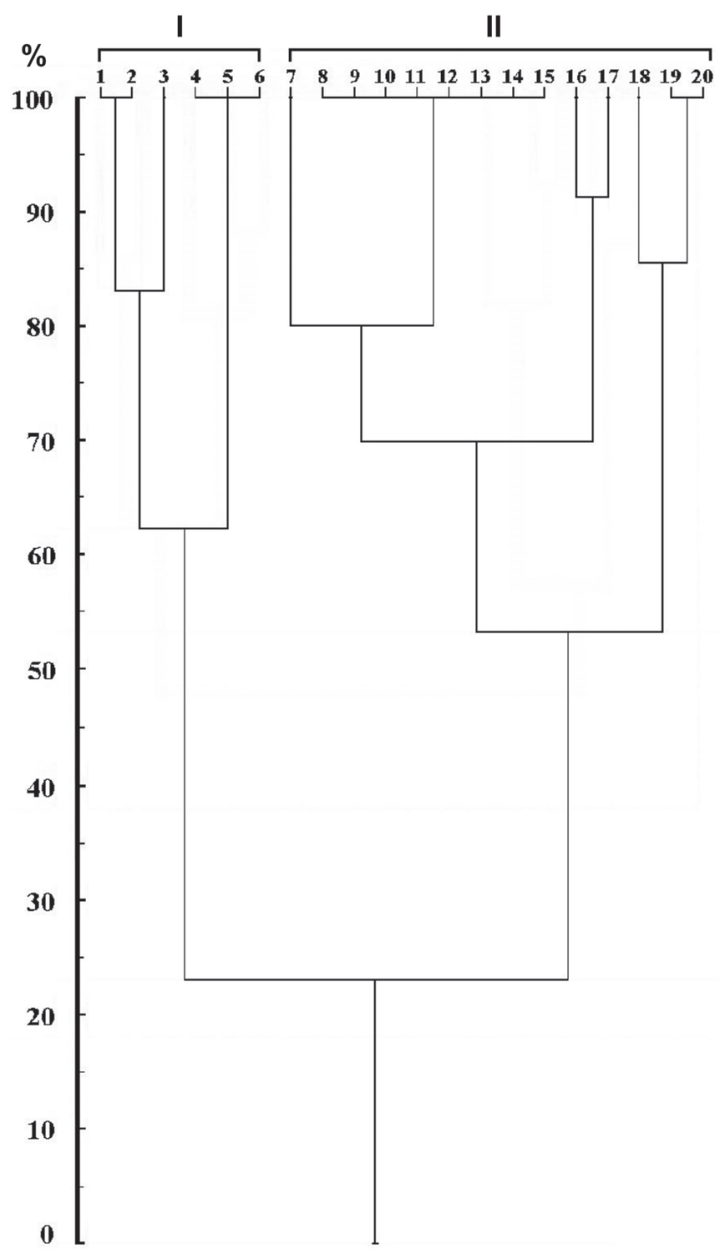

Fig. 4: Dendrograma del índice de afinidad de Jaccard, entre las estaciones del transecto muestreado. Zonas faunísticas de foraminíferos: I y II.

Jaccard's affinity index cluster, between stations of the sampled transect. Foraminiferal zones: I and II.

La Subzona faunística IA se distribuyó entre los 1.80 y $1.50 \mathrm{~m}$ de elevación y comprendió la marisma alta (MA) y media (MM) (estaciones 1-4). Nuevamente la especie dominante fue $H$. manilaensis con una abundancia relativa máxima de un $66 \%$ La Subzona faunística IB se distribuyó entre los 1.35-1.25 m de elevación y coincidió con la marisma baja (MB) (estaciones 5-6). Esta Subzona estuvo codominada por las especies $H$. manilaensis y $M$. fusca con abundancias relativas máximas de 63 y $51 \%$ respectivamente (Fig. 5).

La Subzona faunística IIA se ubicó entre los 1.25-0.45 m de elevación y se distribuyó en parte de la llanura de marea (LM) (estaciones
7-17). Esta Subzona estuvo codominada por las especies aglutinadas $H$. manilaensis y $M$. fusca (abundancias relativas máximas de 28 y $61 \%$ ) y por las especies calcáreas $A$. beccarii y $E$. williamsoni (abundancias relativas máximas de 44 y $28 \%$ ). La Subzona IIB se halló entre los 0.45 y $0 \mathrm{~m}$ de elevación y se distribuyó en la última parte de la llanura de marea (LM) (estaciones 18-20). Esta Subzona estuvo dominada exclusivamente por la especie calcárea $A$. beccarii con una abundancia relativa máxima de $51 \%$ (Fig. 5).

En general la diversidad ( $\left.\mathrm{H}^{\prime}\right)$ fue baja en la Zona I (marisma) y aumentó hacia la Zona II (llanura de marea) con un valor mínimo de 0.417 en la marisma media (MM) y un máximo de 0.909 en la llanura de marea (LM). La riqueza de especies (S) tuvo la misma tendencia, con un mínimo de 5 especies en la Zona I y un máximo de 13 especies en la Zona II (Fig. 6).

Los parámetros salinidad y $\mathrm{pH}$ fueron variables a lo largo del área de estudio. La salinidad fluctuó entre 2.1 y 32.8 , con los valores más altos (30.9-32.8) en las estaciones cercanas al mar (estaciones 18-20) y con los valores más bajos (2.3-2.1) en las estaciones ubicadas en la marisma. Por otro lado el $\mathrm{pH}$ varió entre 6.4 y 7.7; con los valores más bajos (6.4-6.6) en la marisma y los más altos (7.6-7.7) en la llanura de marea (Fig. 6).

\section{DISCUSIÓN}

Un gran número de trabajos han estudiado la distribución vertical de los foraminíferos modernos en las marismas del hemisferio norte (e.g., Le Campion 1970, Phleger 1970, Pujos 1976, Scott \& Medioli 1978, Scott \& Medioli 1980a, Smith et al. 1984, Williams 1989, Patterson 1990, Murray 1991, Jennings \& Nelson 1992, Gehrels 1994a, 1994b, De Rijk 1995, Guilbault et al. 1995, Guilbault et al. 1996, Scott et al. 1996, De Rijk \& Troelstra 1997, Cearreta et al. 2002, Moreno et al. 2005, Woodroffe et al. 2005, Edwards \& Horton 2006, Horton \& Edwards 2006, Fatela et al. 2007, Horton \& Murray 2007, Horton \& Culver 2008, Leorri et al. 2008), no obstante, son escasos los trabajos desarrollados en el hemisferio Sur, substancialmente en las marismas templadas de Chile. 
Todos los autores concuerdan en que estos organismos tienen una distribución vertical dividida en: a) una zona con una comunidad poco diversa, dominada por foraminíferos aglutinados distribuidos en la parte alta y vegetada del intermareal (marisma); y b) una zona con una comunidad más diversa, codominada por especies calcáreas $\mathrm{y}$ aglutinadas, distribuidas en la parte más baja y sin vegetación del intermareal (llanura de marea) (Scott \& Medioli 1980a, Scott et al.
1996, Scott et al. 2001, Woodroffe et al. 2005, Debenay et al. 2006, Semensatto et al. 2009).

Contrariamente, los autores discrepan en el parámetro que controla la distribución de estos organismos en las marismas. Algunos atribuyen el control a la salinidad (e.g., Murray 1971, Patterson 1990, De Rijk 1995, De Rijk \& Troelstra 1997), al pH (e.g., Woodrofe et al. 2005, Fernández \& Zapata 2010), al tamaño del grano en el sedimento (e.g., Matera \& Lee 1972, Scott et al. 1998) o a la zonación floral de
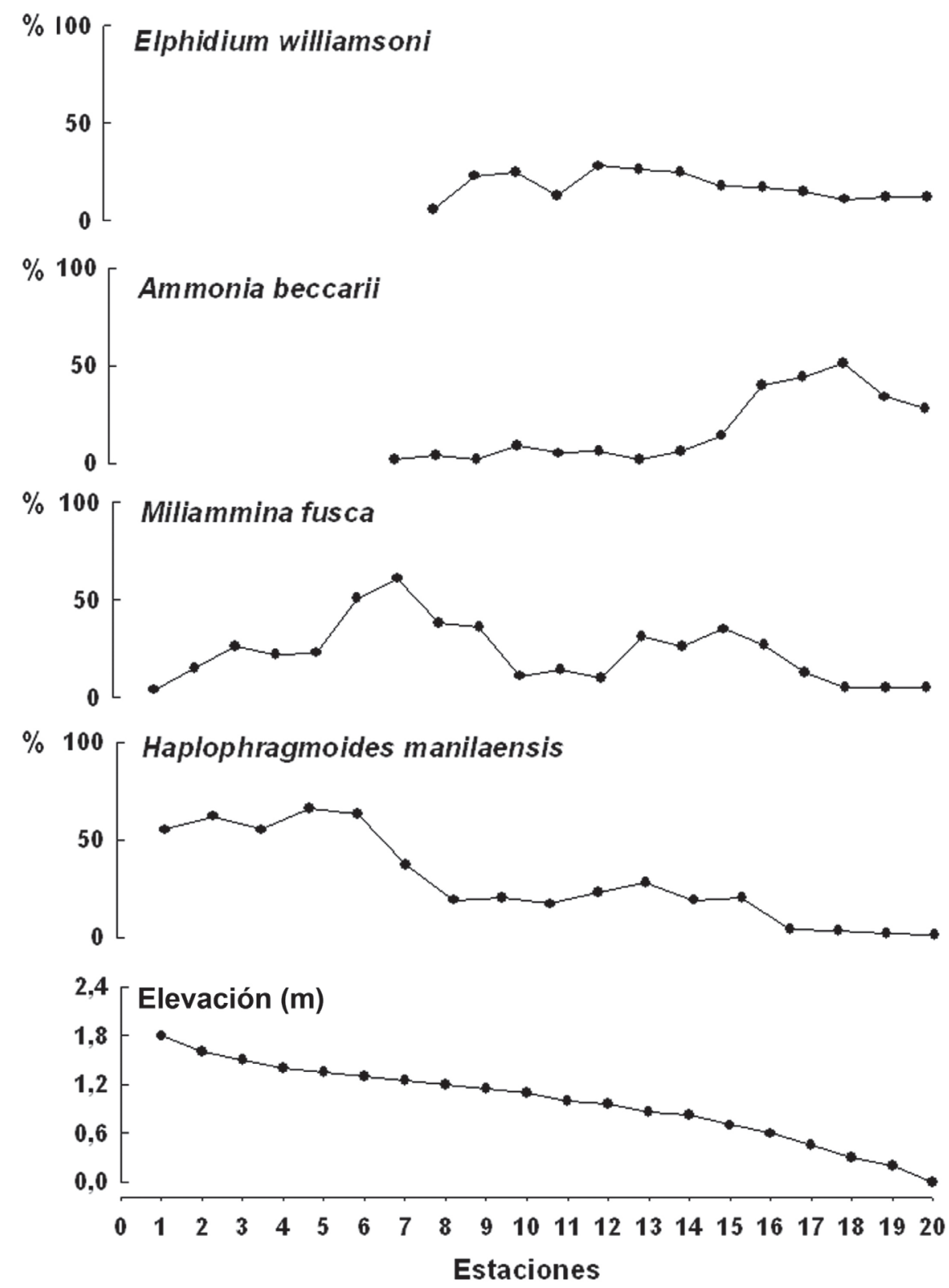

Fig. 5: Elevación y especies dominantes de cada estación en la ensenada de Quillaipe.

Elevation and dominant species of each station in Quillaipe Inlet. 
la marisma (Scott \& Medioli 1978, Scott \& Medioli 1980a, Jennings et al. 1995). No obstante, Horton et al. (1999) indican que la distribución de los foraminíferos está controlada por la elevación del intermareal y la duración de la marea, idea que ha sido consistente con investigaciones posteriores (e.g., Cearreta et al. 2002, Moreno et al. 2005, Edwards \& Horton 2006, Horton \& Edwards 2006, Horton \& Murray 2007) y la base para el desarrollo de la "función de transferencia". Esta conclusión puede explicarse, porque el tamaño del grano, el pH, la materia orgánica y la zonación floral exhiben un importante grado de covarianza con la elevación (Berkeley et al. 2007).

En la ensenada de Quillaipe la distribución vertical de los foraminíferos fue consistente con la registrada en estas investigaciones, reflejando el gradiente marino-continental
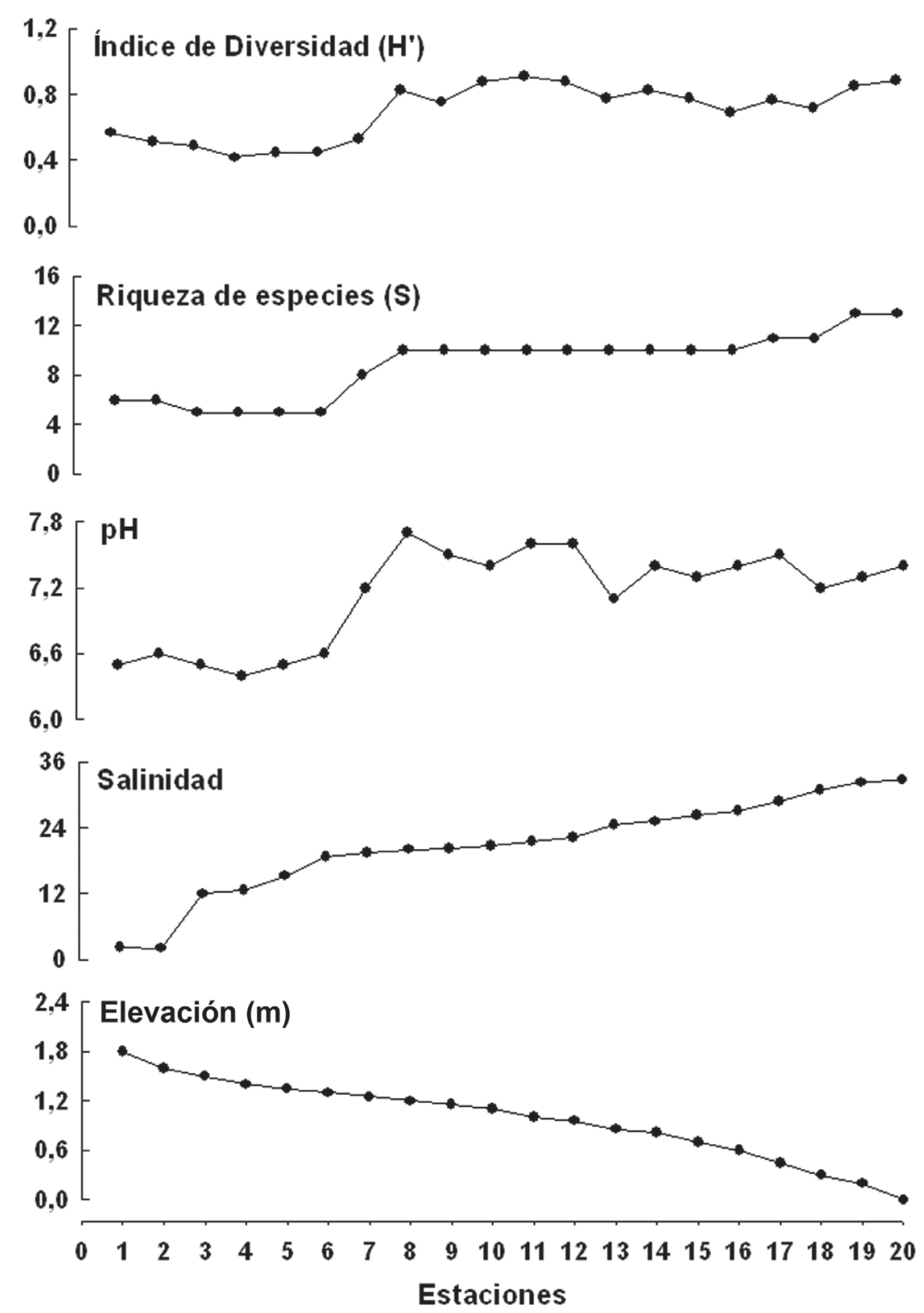

Fig. 6: Índice de diversidad, riqueza de especies, pH, salinidad y elevación de cada estación en la ensenada de Quillaipe.

Diversity index, species richness, $\mathrm{pH}$, salinity and elevation of each station in Quillaipe Inlet. 
generalizado a nivel mundial. En primer lugar se registró una comunidad con baja diversidad, dominada por especies aglutinadas y restringida a la marisma (Zona I) y en segundo lugar una comunidad con una diversidad más alta, codominada por especies calcáreas y aglutinadas distribuidas en la llanura de marea (Zona II). Esta zonación es corroborada por el análisis de similitud e ilustrada por el dendrograma.

\section{Diversidad de especies}

Si bien los valores de diversidad obtenidos en este estudio son bajos cerca del mar $\left(\mathrm{H}^{\prime}<1.0\right)$ y aún más bajos hacia el continente $\left(\mathrm{H}^{\prime}<0.5\right)$, son propios de un ambiente parálico como el estudiado y reflejan el alto estrés al que está expuesto. Por ejemplo Buzas \& Gibson (1969) indican que un ambiente marino tiene usualmente valores de diversidad $\left(\mathrm{H}^{\prime}\right)$ mayores a 3.0 y que valores menores son propios de ambientes de alta inestabilidad. Por otro lado, Murray (1991) y Horton \& Murray (2007) indican valores de diversidad $\left(\mathrm{H}^{\prime}\right)$ menores a 2.1 como típicos de ambientes parálicos con baja salinidad. Estos resultados son congruentes con los obtenidos en este estudio.

\section{Especies dominantes}

La composición de la microfauna de las marismas salobres suele ser similar a nivel mundial, debido a que en todos los ambientes parálicos predomina un alto estrés ambiental (Debenay 2000). No obstante, la importancia de estudiar la distribución de estos organismos en distintos puntos geográficos tiene fundamento en que la historia geológica entre sitios es muy distinta, por lo que también lo serán las especies características (Boltovskoy 1976). En este estudio la Zona I, que corresponde a la marisma, estuvo dominada por la especie aglutinada $H$. manilaensis y la Zona II, que se corresponde con la llanura de marea, estuvo dominada principalmente por la especie calcárea $A$. beccarii. Adicionalmente, considerando un $65 \%$ de afinidad es posible identificar dos subzonas (IA, IB y IIA, IIB) para ambas Zonas.

La Subzona faunística IA estuvo dominada por $H$. manilaensis y se correspondió con la marisma alta y media. La Subzona IB estuvo codominada por $H$. manilaensis y $M$. fusca y coincidió con la marisma baja. Por otro lado, la Subzona IIA se distribuyó en la parte distal de la llanura de marea y estuvo codominada por las especies aglutinadas $H$. manilaensis y $M$. fusca y por las calcáreas $A$. beccarii y $E$. williamsoni. Finalmente, la Subzona IIB se distribuyó en la última parte de la llanura de marea y estuvo dominada exclusivamente por la especie calcárea $A$. beccarii.

Otros estudios coinciden o difieren con nuestros resultados. Williams (1989) indica a $T$. inflata como la especie dominante en la marisma alta y media en Columbia Británica, Canadá. Fatela et al. (2007) indican en Portugal a $T$. inflata como especie dominante de la marisma alta del estuario Lima y a $H$. manilaensis como especie dominante de la marisma alta del estuario Minho. En cambio, Scott et al. (1990) indican a Ammotium salsum (Cushman \& Brönnimann, 1948) como el taxa dominante en una marisma alta de Estados Unidos. Horton \& Edwards (2005), en base a una investigación realizada en 13 marismas de Inglaterra, señalan como especies dominantes de la marisma alta y media a $J$. macrescens, $T$. inflata, M. fusca y Haplophragmoides spp. No obstante, nunca codominaron todas las especies en un mismo sitio. De igual forma, Horton \& Edwards (2005) indican a M. fusca y Quinqueloculina spp. dominando en la marisma baja. Pero Fatela et al. (2007), indica como especie dominante para esta subzona a Psammosphera spp. Por otro lado, casi todos los estudios reconocen en la llanura de marea un dominio de $A$. beccarii asociada a Elphidium spp. (Debenay \& Guillou 2002).

Los resultados entregados por estas investigaciones, principalmente los de Horton \& Edwards (2005) y Fatela (2007), demuestran que las especies dominantes de las marismas no son extrapolables a los ambientes parálicos de otros puntos geográficos e incluso dentro de una misma localidad.

\section{Parámetros y su significado}

Numerosos parámetros controlan la ecología de los foraminíferos bentónicos a diferentes escalas. Estos se pueden dividir en aquellos que afectan principalmente los patrones de distribución y en los que afectan mayormente la abundancia (Horton \& Murray 2007). 
La elevación, si bien no es un verdadero "parámetro", es determinante durante los periodos de pleamar, creando diferencias en las condiciones hidrológicas y hialinas a través del intermareal. Pero en algunas ocasiones, la salinidad también puede variar independientemente de la elevación y ser afectada por la micromorfología de la marisma, el tiempo de exposición al régimen de marea, la precipitación e inundación por agua dulce (Charman et al. 2002), causas que podrían ser el origen de su irregularidad en el intermareal de Quillaipe. Aún así, los valores más altos se ubicaron claramente en las estaciones cercanas al mar (llanura de marea) y los más bajos en las estaciones ubicadas en la marisma.

Por otro lado, la salinidad afectó la composición y distribución de la microfauna, restringiendo las especies más estenohalinas a la llanura de marea. Estas especies son de tipo calcáreo, las que son altamente dependientes de la salinidad ya que construyen sus caparazones de carbonato de calcio (Boltovskoy 1965).

Igualmente, se registraron especies eurihalinas, donde la más representativa fue la aglutinada $M$. fusca, foraminífero capaz de soportar grandes variaciones de salinidad y recurrente en ambientes salobres como estuarios, lagunas costeras y marismas (Boltovskoy et al. 1980).

En general, las especies aglutinadas tienen distribuciones amplias en los ambientes parálicos, debido a que construyen sus caparazones aglutinando material del sustrato y usan el carbonato de calcio tan sólo como sustancia cementante, por lo que dependen menos de la salinidad (Boltovskoy 1965). Por otro lado, en comparación a otras especies calcáreas, A. beccarii tuvo una distribución amplia, pero restringida únicamente a la llanura de marea. Esto sugiere que en esta y otras especies, la osmorregulación juega un rol importante (Debenay 2000).

Paralelamente, algunos autores (Scott \& Medioli 1978, Scott \& Medioli 1980a, Jennings et al. 1995) han propuesto que la distribución vertical de los foraminíferos está controlada por la zonación floral que presentan las marismas (marisma alta, media y baja). En este estudio no existió relación entre las zonaciones florales y la distribución de los foraminíferos. El índice de similitud permitió identificar que la vegetación solo restringe la distribución de los foraminíferos calcáreos lo que puede ser consecuencia de los bajos valores de salinidad y $\mathrm{pH}$ registrados en este punto. Esta idea es concordante con los resultados de otras investigaciones, las que han indicado que el rol más importante de la vegetación es solo entregar protección, alimento y contribuir a disminuir el $\mathrm{pH}$ en la interface sedimento-agua (Duchemin et al. 2005, Horton \& Murray 2007).

El pH es otro parámetro ecológico que influye estrechamente en la composición de la microfauna (Murray 2006). Los valores bajos de $\mathrm{pH}$ afectan negativamente a las especies calcáreas (Murray \& Alve 1999), pero no tienen un mayor efecto sobre los foraminíferos aglutinados, porque el tipo de construcción de sus caparazones les otorga una mayor resistencia a la disolución (Phleger 1966, Bradshaw 1968).

En este estudio, los valores más bajos de $\mathrm{pH}$ fueron registrados en la marisma, lo que se debe a que en ese ambiente existe un alto flujo de materia orgánica, que al ser oxidada, incrementa la producción de ácido carbónico, nítrico y sulfúrico (Schafer 2000).

El efecto del $\mathrm{pH}$ sobre la conservación y la distribución de los foraminíferos calcáreos ha sido registrado anteriormente en la ensenada Quillaipe por Fernández \& Zapata (2010). Los autores registraron caparazones de $A$. beccarii con distintos grados de disolución, concluyendo que esto estaba estrechamente relacionado con los valores de $\mathrm{pH}$ medidos in situ y con el tiempo de exposición a los distintos rangos de este parámetro. De igual forma, concluyeron que el $\mathrm{pH}$ determina la distribución vertical y horizontal de este foraminífero calcáreo en el intermareal, restringiéndolo en consecuencia solo a la llanura de marea. Sus resultados fueron consistentes con los de este y otros estudios (Scott \& Medioli 1980a, Jonasson \& Patterson 1992, Murray \& Alve 1999).

\section{Especies indicadoras del máximo nivel del mar}

Una contribución importante, al analizar la distribución vertical de los foraminíferos, es identificar las especies que ayudan a delimitar el nivel máximo de la pleamar. 
Diversas investigaciones permiten concluir que la distribución de estos organismos es controlada por distintos factores locales. No obstante, todos exhiben un importante grado de covarianza con la elevación (Berkeley et al. 2007). Por lo mismo, el significado indicativo de los foraminíferos es comúnmente expresado en relación a la elevación de la marisma (Horton et al. 1999).

Williams (1989) señaló a T. inflata como el componente principal de la marisma alta del río Fraser, Columbia Británica. Un estudio posterior desarrollado en la misma área por Patterson (1990), reveló a J. macrescens como la especie principal de la marisma alta. Scott et al. (1980), indicaron como habitante de la marisma alta y como indicador del nivel más alto del mar a A. salsum en una marisma de Estados Unidos. Contrariamente, Jennings \& Nelson (1992) indicaron a $M$. fusca como el componente principal de la marisma alta en Oregon, Estados Unidos. Coles \& Funnell (1981) estudiaron el sector de Broadlands, Inglaterra e identificaron un codominio de $J$. macrescens y T. inflata en la marisma alta. Análogamente, Boomer (1998) y Funnell \& Boomer (1998) en North Norfolk, Inglaterra y Murray (1991) en Europa señalaron la misma asociación para la marisma alta. En cambio Jennings et al. (1995) y Southall et al. (2006) indicaron que solo T. salsa se restringió a la marisma alta. Por el contrario, Scott \& Medioli (1980a), Guilbault et al. (1995), Ozarko et al. (1997) y Vázquez et al. (2007), señalaron que solo J. macrescens se restringió a la marisma alta y define bien el nivel máximo de la pleamar.

En este estudio la asociación T. salsa y $J$. macrescens, no fue dominante pero sí se restringió a la marisma alta y a bajos valores de salinidad y pH. En consecuencia, estas dos especies podrían ser usadas como indicadoras del máximo nivel de la pleamar en la ensenada de Quillaipe. Lamentablemente, el límite superior de la marisma está interrumpido por una carretera, lo que imposibilita determinar si es una especie o la asociación de ambas la más apropiada para este fin.

\section{Especies exóticas}

Las especies registradas en el área estudiada pueden ser divididas en indígenas o exóticas. Las indígenas son aquellas que se encontraron en todo el intermareal y son características de ambientes parálicos (e.g., A. beccarii, Elphidium spp.). En cambio, las especies exóticas son aquellas que son infrecuentes en el área estudiada pero comunes en el litoral chileno (Zapata \& Cea 2004). Su presencia puede deberse a la fuerza de arrastre que ejerce la corriente de marea desde el mar abierto hacia la ensenada de Quillaipe. Entre estas especies podemos mencionar a Bolivina doniezi Cushman \& Wickenden, 1929; Cibicides aknerianus (Orbigny, 1846) y Discorbis williamsoni Chapman \& Parr, 1932.

\section{CONCLUSIONES}

Los resultados obtenidos en este trabajo nos permiten concluir que la distribución vertical de los foraminíferos de la ensenada de Quillaipe es consistente con el gradiente marino-continental generalizado a nivel mundial. De igual forma, se distribuyen en relación a la salinidad, $\mathrm{pH}$ y elevación de la marisma, pero nunca en concordancia a la zonación floral de la marisma.

Por otro lado la asociación aglutinada $T$. salsa y $J$. macrescens, se restringió a la marisma alta y a bajos valores de salinidad y $\mathrm{pH}$ por lo que estas dos especies podrían ser usadas como indicadoras del máximo nivel de la pleamar en la ensenada de Quillaipe. No obstante, no es posible determinar si una o la asociación de ambas especies es la más apropiada para este fin.

Esto nos permite afirmar que es factible la aplicación de la "función de transferencia" en la marisma templada de la ensenada de Quillaipe.

Finalmente se sugiere realizar en un futuro muestreos estacionales de los parámetros salinidad, $\mathrm{pH}$, sedimento, fluctuaciones mareales y de la foraminiferofauna lo que permitirá decretar con completa certeza las especies apropiadas para delimitar a pequeña escala zonas verticales que se correspondan con las alturas de marea y los parámetros que mejor determinan sus distribuciones.

\section{AGRADECIMIENTOS}

Los autores agradecen a la Dirección de Investigación de la Universidad de Los Lagos por el 
financiamiento de la investigación, al personal del Laboratorio de Microscopía Electrónica de la Universidad de Concepción por su buena disposición para el fotografiado de las especies de foraminíferos y a tres revisores anónimos que con sus comentarios sobre la versión preliminar contribuyeron a mejorar el manuscrito. El primer autor agradece especialmente a Alfredo Dawson por la revisión de los textos en inglés y a Fabiola Barrientos Loebel por la edición de las figuras y el constante e incondicional apoyo entregado durante el desarrollo de esta investigación.

\section{LITERATURA CITADA}

ALVE E \& J MURRAY (1999) Marginal marine environments of the Skagerrak and Kattegat: A baseline study of living (stained) benthic foraminiferal ecology. Palaeogeography, Palaeoclimatology, Palaeoecology 146: 171-193.

BERKELEY A, CT PERRY, SG SMITHERS, BP HORTON \& KG TAYLOR (2007) A review of the ecological and taphonomic controls on foraminiferal assemblage development in intertidal environments. Earth Science Reviews 83: 205-230.

BOLTOVSKOY E (1965) Los foraminíferos recientes. EUDEBA, Buenos Aires, Argentina.

BOLTOVSKOY E (1976) Distribution of recent foraminifera of the South America region. Academia Press, London, England.

BOLTOVSKOY E \& F THEYER (1970) Foraminíferos recientes de Chile central. Revista del Museo Argentino de Ciencias Naturales Bernardino Rivadavia, Hidrobiologia 2: 279-379.

BOLTOVSKOY E, G GIUSSANI, S WATANABE \& R WRIGHT (1980) Atlas of benthic shelf foraminifera of the southwest Atlantic. Dr. W. Junk by Publications. The Hague, Boston, London.

BOOMER I (1998) The relationship between meiofauna (Otracoda, Foraminifera) and tidal levels in modern intertidal environments of North Norfolk: A tool for a palaeoenvironmental reconstruction. Bulletin of Geological Society of Norfolk 46: 17-29.

BRADSHAW J (1968) Environmental parameters and marsh foraminifera. Limnology and Oceanography 13: 26-38.

BUZAS MA \& TG GIBSON (1969) Species diversity: Benthonic foraminifera in western North Atlantic. Science 163: 72-75.

CEARRETA A, MJ IRABIEN, I ULIBARRI, I YUSTA, IW CROUDACE \& AB CUNDY (2002) Recent salt marsh development and natural regeneration of reclaimed areas in the Plentzia estuary, N. Spain. Estuarine, Coastal and Shelf Science 54: 863-886.

CHARMAN DJ, HM ROE \& WR GEHRELS (2002) Modern distribution of saltmarsh testate amoebae: Regional variability of zonation and response to environmental variables. Journal of Quaternary Science 17: 387-409.

COLES BPL \& BM FUNNELL (1981) Holocene palaeoenvironments of Broadland, England. Special Publication International Association of Sedimentologists 5: 123-131.
CRISCI J \& M LÓPEZ (1983) Introducción a la teoría y práctica de la taxonomía numérica. OEA, Serie de Biología, Monografía 26: 1-132.

CUSMINSKY GC, E BERNASCONI \& L CALVOMARCILESE (2009) Holocene benthic foraminífera from Bahía Blanca estuary: A review and update of systematic and palaeoenvironmental aspects. Holocene 19: 12211231.

DEBENAY JP (2000) Foraminifers of paralic tropical environments. Micropaleontology 46: 153-160.

DEBENAY JP \& J GUILLOU (2002) Ecological transitions indicated by foraminiferal assemblages in paralic environments. Estuaries 25: $1107-1120$.

DEBENAY JP, E BICCHI, E GOUBERT \& E CHÂTELET (2006) Spatio-temporal distribution of benthic foraminifera in relation to estuarine dynamics (Vie Estuary, Vendée, France). Estuarine, Coastal and Shelf Science 67: 181-197.

DEBENAY JP, BB EICHLER, W DULEBA, C BONETTI \& P EICHLER-COELHO (1998) Water stratification in coastal lagoons: Its influence on foraminiferal assemblages in two Brazilians lagoons. Marine Micropaleontology 35: 67-89.

DE RIJK S (1995) Salinity control on the distribution of salt-marsh foraminifera (Great-Marshes, Massachusetts). Journal of Foraminiferal Research 25: 156-166.

DE RIJK \& SR TROELSTRA (1997) Saltmarsh foraminifera from the Great Marshes, Massachusetts: Environmental controls. Palaeogeography, Palaeoclimatology, Palaeoecology 130: 81-112.

DUCHEMIN G, FJ JORISSEN, F REDOIS \& JP DEBENAY (2005) Foraminiferal microhabitats in a high marsh: Consequences for reconstructing past sea levels. Palaeogeography, Palaeoclimatology, Palaeoecology 226: 167-185.

EDWARDS RJ \& BP HORTON (2006) Developing detailed records of relative sea-level change using a foraminiferal transfer function: An example from North Norfolk, UK. Philosophical transactions 364: 973-991.

EDWARDS RJ, AJ WRIGHT \& O VAN DE PLASSCHE (2004) Surface distribution of salt-marsh foraminifera from Connecticut, USA: Modern analogues for high-resolution sea level studies. Marine Micropaleontology 51: 1-21.

EMERY K (1961) A simple method of measuring beach profiles. Limnology and Oceanography 6: 90-93.

FATELA F, J MORENO \& C ANTUNES (2007) Salinity influence on foraminiferal tidal marsh assemblages of NW Portugal: An anthropogenic constraint? Thalassas 23: 51-63.

FERNÁNDEZ L \& J ZAPATA (2010) Registro tafonómico de Ammonia beccarii (Linné, 1758) (Protozoa: Foraminiferida) en la ensenada Quillaipe $\left(41^{\circ} 32^{\prime}\right.$ S; $72^{\circ} 44^{\prime}$ O), Chile. Latin American Journal of Aquatic Research 38: 286291.

FUNNELL BM \& I BOOMER (1998) Microbiofacies tidal-level and age deduction in Holocene saltmarsh deposits on the North Norfolk Coast. Bulletin of Geological Society of Norfolk 46: 3155.

GEHRELS WR (1994a) Determining relative sea-level 
change from salt-marsh foraminifera and plant zones on the coast of Maine, USA. Journal of Coastal Research 10: 990-1009.

GEHRELS WR (1994b) Holocene sea-level changes in the northern Gulf of Maine: Regional trends and local fluctuations determined from foraminiferal analyses and paleotidal modeling. Ph.D. Thesis, Maine University, Orono, USA.

GEHRELS WR, HM ROE \& DJ CHARMAN (2001) Foraminifera, testate amoebae and diatoms as sea-level indicators in UK saltmarshes: A quantitative multiproxy approach. Journal of Quaternary Science 16: 201-220.

GOLDSTEIN ST \& EB HARBEN (1993) Taphofacies implications of infaunal foraminiferal assemblages in a Georgia salt marsh, Sapelo Island. Micropaleontology 39: 53-62.

GOLDSTEIN ST \& GT WATKINS (1998) Elevation and the distribution of salt-marsh foraminifera, St. Catherine's Island, Georgia: A taphonomic approach. Palaios 13: 570-580.

GUILBAULT J, J CLAGUE \& M LAPOINTE (1995) Amount of subsidence during late Holocene earthquake-evidence from fossil tidal marsh foraminifera at Vancouver Island, west coast of Canada. Palaeogeography, Palaeoclimatology, Palaeoecology 11: 49-71.

GUILBAULT J, J CLAGUE \& M LAPOINTE (1996) Foraminiferal evidence for the amount of coseismic subsidence during a late Holocene earthquake on Vancouver Island, west coast of Canada. Quaternary Sciences Review 15: 913937.

HAYWARD B, H GRENFELL \& C REID (1997) Foraminiferal associations in Wanganui Bight and Queen Charlotte Sound, New Zealand. New Zealand Journal of Marine and Freshwater Research 31: 337-365.

HAYWARD B, H GRENFELL, C REID \& K HAYWARD (1999) Recent New Zealand shallow-water benthic foraminifera: Taxonomy, ecologic distribution, biogeography, and use in paleoenvironmental assessment. Institute of Geological and Nuclear Science Monograph 16: 1-166.

HORTON BP (1999) The distribution of contemporary intertidal foraminifera at Cowpen Marsh, Tees Estuary, UK: Implications for studies of Holocene sea-level changes. Palaeogeography, Palaeoclimatology, Palaeoecology 149: 127-149.

HORTON BP \& S CULVER (2008) Modern intertidal foraminifera of the outer banks, North Carolina, U.S.A., and their applicability for sea-level studies. Journal of Coastal Research 24: 11101125.

HORTON BP \& RJ EDWARDS (2003) Seasonal distributions of foraminifera and their implications for sea-level studies. SEPM Society for Sedimentary Geology Special Publication 75: 21-30.

HORTON BP \& RJ EDWARDS (2005) The application of local and regional transfer function to the reconstruction of Holocene sea levels, North Norfolk, England. The Holocene 15: 216-228.

HORTON BP \& RJ EDWARDS (2006) Quantifying Holocene sea level change using intertidal Foraminifera: Lessons from the British Isles. Cushman Foundation of Foraminiferal Research, Special Publication 40: 1-97.
HORTON B \& J MURRAY (2007) The roles of elevation and salinity as primary control son living foraminiferal distributions: Cowpen Marsh, Tees Estuary, UK. Marine Micropaleontology 63: 169186.

HORTON BP, RJ EDWARDS \& JM LLOYD (1999) UK intertidal foraminiferal distributions: Implications for sea-level studies. Marine Micropaleontology 36: 205-223.

HORTON BP, P LARCOMBE, SA WOODROFFE, JE WHITTAKER, MR WRIGHT \& C WYNN (2003) Contemporary foraminiferal distributions of a mangrove environment, Great Barrier Reef coastline, Australia: Implications for sea-level reconstruction. Marine Geology 198: 225-243.

HORTON BP, JE WHITTAKER, KH THOMPSON, MIJ HARDBATTLE, A KEMP, SA WOODROFFE \& MR WRIGHT (2005) The development of a modern foraminiferal data set for sea-level reconstruction, Wakatobi Marine National Park, Southeast Sulawesi, Indonesia. Journal of Foraminiferal Research 35: 1-14.

JENNINGS A \& A NELSON (1992) Foraminiferal assemblage zones in Oregon tidal marshesrelation to marsh floral zones and sea level. Journal of Foraminiferal Research 22: 13-29.

JENNINGS A, A NELSON, D SCOTT \& J ARAVENA (1995) Marsh foraminiferal assemblages in the Valdivia estuary, south-central Chile, relative to vascular plants and sea level. Journal Coastal Research 11: 107-123.

JONASSON K \& R PATTERSON (1992) Preservation potential of marsh benthic foraminifera from the Fraser River delta, British Columbia. Micropaleontology 38: 289-301.

KRAUSE G (2004) The "Emery-Method" revisitedperformance of an inexpensive method of measuring beach profiles and modifications. Journal Coastal Research 20: 340-346.

LE CAMPION J (1970) Contribution à l' etudes des foraminifères du Bassin d'Arcachon et du proche ocean. Bulletin de l' Institut de Géologie du Bassin d' Aquitaine 8: 3-98.

LEORRI E, BP HORTON \& A CEARRETA (2008) Development of a foraminifera-based transfer function in the Basque marshes, N. Spain: Implications for sea-level studies in the Bay of Biscay. Marine Geology 251: 60-74.

LOEBLICH A \& H TAPPAN (1988) Foraminiferal genera and their classification. Van Nostrand, New York, USA.

MATERA NJ \& JJ LEE (1972) Environmental factors affecting standing crop of foraminifera in sublittoral and psammolittoral communities of a Long-Island saltmarsh. Marine Biology 14: 89103.

MORENO J, F FATELA, C ANDRADE, J CASCALHO, F MORENO \& T DRAGO (2005) Living foraminiferal assemblages from the Minho and Coura estuaries (Northern Portugal): A stressfull environment. Thalassas 21: 17-28.

MURRAY J (1971) Living foraminiferids of tidal marshes: A review. Journal of Foraminiferal Research 1: 153-161.

MURRAY J (1991) Ecology and palaeoecology of benthic foraminifera. Longman, Harlow, England.

MURRAY J (2000) The enigma of the continued use of total assemblages in ecological studies of 
benthic foraminifera. Journal of Foraminiferal Research 30: 244-245.

MURRAY J (2006) Ecology and applications of benthic foraminifera. Cambridge University Press, Cambridge.

MURRAY J \& E ALVE (1999) Natural dissolution of modern shallow water benthic foraminifera: Taphonomic affects on the palaeoecological record. Palaeogeography, Palaeoclimatology, Palaeoecology 146: 195-209.

OZARKO D, R PATTERSON \& H WILLIAMS (1997) Marsh foraminifera from Nanaimo, British Columbia: Infaunal habitat and taphonomic implications. Journal of Foraminiferal Research 27: 51-68.

PASCUAL A \& J RODRÍGUEZ-LÁZARO (2006) Marsh development and sea level changes in the Gernika Estuary (Southern Bay of Biscay): Foraminifers as tidal indicators. Scientia Marina 70: 101-117.

PATTERSON R (1990) Intertidal benthic foraminiferal biofacies on the Fraser River delta, British Columbia: Modern distribution and palaeoecological importance. Micropaleontology 36: 183-199.

PATTERSON R, W GEHRELS, D BELKNAP \& A DALBY (2004) The distribution of salt marsh foraminiferal at Little Dipper Harbour New Brunswick, Canada: Implications for development of widely applicable transfer functions in sea-level research. Quaternary International 120: 185-194.

PHLEGER F (1966) Patterns of living marsh foraminifera in South Texas coastal lagoons. Boletín de la Sociedad Geológica Mexicana 28: 1-44.

PHLEGER F (1970) Foraminiferal populations and marine marsh processes. Limnology and Oceanography 15: 522-534.

PUJOS M (1976) Ecologie des foraminifères benthiques et des thècamoebiens de la Gironde et au plateau continental SudGascogne. Application à la connaissaance du Quaternaire terminal de la región Ouest-Gironde. Mem. Bulletin de l' Institut de Géologie du Bassin d' Aquitaine 8: 1-274.

SAFFERT H \& E THOMAS (1998) Living foraminifera and total populations in salt marsh peat cores: Kelsey Marsh (Clinton, CT) and The Great Marshes (Barnstaple, MA). Marine Micropaleontology 33: 175-202.

SÁIZ F \& V AVENDAÑO (1976) Análisis comunitario e instrumentos para su interpretación en artrópodos del Parque Nacional "Fray Jorge". Anales del Museo de Historia Natural de Valparaíso 9: 89-104.

SAN MARTÍN C \& C RAMÍREZ (2002) Sinecología de una marisma en el Seno de Reloncaví (Llanquihue, X Región, Chile). Revista Geográfica de Valparaíso 22: 307-319.

SCHAFER C (2000) Monitoring nearshore marine environments using benthic foraminifera: Some protocols and pitfalls. Micropaleontology 46: 161-169.

SCOTT DB (2006) Testate rhizopods as reliables, costeffective indicators of environmental change. Anuário do Instituto de Geociências 29: 129-140.

SCOTT DB \& R LECKIE (1990) Foraminiferal zonation of Great Sippewissett salt marsh (Falmouth, Massachusetts). Journal of Foraminiferal Research 20: 248-266.

SCOTT DB \& F MEDIOLI (1978) Vertical zonation of marsh foraminifera as accurate indicators of former sea levels. Nature 272: 528-531.

SCOTT DB \& F MEDIOLI (1980a) Quantitative studies of marsh foraminifera distribution in Nova Scotia: Implications for sea-level studies. Cushman Foundation for Foraminiferal Research, Special Publication 17: 1-58.

SCOTT DB \& F MEDIOLI (1980b) Living $\mathrm{v} / \mathrm{s}$ total foraminiferal populations: Their relative usefulness in paleoecology. Journal of Paleontology 54: 814-831.

SCOTT DB, E COLLINS, J DUGGAN, A ASIOLI, T SAITO \& S HASEGAWA (1996) Pacific Rim marsh foraminiferal distributions: Implications for sea-level studies. Journal of Coastal Research 12: 850-861.

SCOTT DB, F MEDIOLI \& R BRAUND (2003) Foraminifera from the Cambrian of Nova Scotia: The oldest multichambered foraminifera. Micropaleontology 49: 109-126.

SCOTT DB, F MEDIOLI \& C SCHAFER (2001) Monitoring of coastal environments using foraminifera and thecamoebian indicators. Cambridge University Press, New York.

SCOTT DB, CT SCHAFER \& FS MEDIOLI (1980) Eastern Canada estuarine foraminifera: A framework for comparison. Journal of Foraminiferal Research 10: 205-234.

SCOTT G, L THOMPSON, R HITCHIN \& J SCOURSE (1998) Observations on selected salt-marsh and shallow-marine species of agglutinated foraminifera: Grain size and mineralogical selectivity. Journal of Foraminiferal Research 28 : 261-267.

SEMENSATTO D, R FERREIRA, D DIAS-BRITO \& C COELHO-JR (2009) Foraminiferal ecological zonation along a Brazilian mangrove transect: Diversity, morphotypes and the influence of subaerial exposure time. Revue de Micropaléontologie 52: 67-74.

SHANNON E \& W WIENER (1963) The mathematical theory of communication. University of Illinois Press, Urbana, USA.

SHOA (2007) Tabla de marea de la Armada de Chile. Servicio Hidrográfico y Oceanográfico de la Armada de Chile.

SMITH DA, DB SCOTT \& FS MEDIOLI (1984) Marsh foraminifera in the Bay of Fundy: Modern distribution and application to sea-level determinations. Maritime Sediments Atlas Geology 20: 127-142.

SOUTHHALL K, W GEHRELS \& B HAYWARD (2006) Foraminifera in a New Zealand salt marsh and their suitability as sea-level indicators. Marine Micropaleontology 60: 167-179.

VÁZQUEZ N, A BABALOLA, R BOUDREAU, R PATTERSON, H ROE \& C DOHERTY (2007) Modern distribution of salt marsh foraminifera and thecamoebians in the Seymour-Belize Inlet complex, British Columbia, Canada. Marine Geology 242: 39-63.

VÁZQUEZ N \& R PATTERSON (2008) An illustrated guide to fjord foraminifera from the SeymourBelize inlet complex, Northern British 
Columbia, Canada. Paleontología Electrónica 11: 45 (online) URL: http://palaeo-electronica.org/ 2008_1/145/index.html (accedido Enero 1, 2010)

WILLIAMS HFL (1989) Foraminiferal zonations on the Fraser River delta and their application paleoenvironmental Palaeogeography, interpretations. Palaeoecology 73: 39-50

WOODROFFE S, B HORTON, P LARCOMBE \& J
WHITTAKER (2005) Intertidal mangrove foraminifera from the central great Barrier Reef Shelf, Australia: Implications for sea-level reconstruction. Journal of Foraminiferal Research 35: 259-270.

ZAPATA J \& C CEA (2004) Foraminíferos litorales recientes del norte de Chile. Boletín de la Sociedad de Biología de Concepción (Chile) 75: 7-58.

Editor Asociado: Humberto E. González

Recibido el 29 de enero de 2010; aceptado el 10 de septiembre de 2010 
